\title{
Diagnostic accuracy of fine needle aspiration cytology in providing a diagnosis of cervi- cal lymphadenopathy among HIV-infected patients
}

\author{
David Muyanja ${ }^{1,2}$, Robert Kalyesubula ${ }^{1,3,4}$, Elizabeth Namukwaya ${ }^{1,3,5}$, \\ Emmanuel Othieno ${ }^{3,6}$, Harriet Mayanja-Kizza $^{1}$
}

1. School of Medicine, Makerere University College of Health Sciences, Kampala, Uganda

2. Mengo Hospital, Department of Medicine, Kampala, Uganda

3. Mulago National Referral Hospital, Kampala, Uganda

4. Department of Physiology, School of Biomedical Sciences, Makerere University College of Health Sciences, Kampala, Uganda

5. Mulago Palliative Care Unit, Kampala, Uganda

6. Department of Pathology, Makerere University College of Health Sciences, Kampala, Uganda.

\begin{abstract}
Background: Opportunistic infections and malignancies cause lymphadenopathy in HIV-infected patients. The use and accuracy of fine needle aspiration cytology in diagnosing of cervical lymphadenopathy among HIV-infected patients is not well studied in Uganda.

Objective: The aim of this study was to determine the diagnostic accuracy of fine needle aspiration cytology in providing a diagnosis of cervical lymphadenopathy among HIV-infected patients in Uganda.

Methods: We consecutively recruited adult HIV-infected patients with cervical lymphadenopathy admitted to Mulago Hospital medical wards. Clinical examination, fine needle aspiration and lymph node biopsy were performed. We estimated the sensitivity, specificity; negative and positive predictive values using histology as the gold standard.

Results: We enrolled 108 patients with a mean age of 33 years (range, 18-60), 59\% were men and mean CD4 was 83(range, $22-375)$ cells $/ \mathrm{mm}^{3}$. The major causes of cervical lymphadenopathy were: tuberculosis (69.4\%), Kaposi's sarcoma-KS (10.2\%) and reactive adenitis $(7.4 \%)$. Overall fine needle aspiration cytology accurately predicted the histological findings in 65 out of 73 cases $(89 \%)$ and missed 7 cases $(9.5 \%)$. With a sensitivity of $93.1 \%$, specificity of $100 \%$, positive predictive value of $100 \%$ and negative predictive value of $78.7 \%$ for tuberculosis and $80 \% ; 98.4 \% ; 88.9 \%$ and $98.9 \%$ for KS respectively. No fine needle aspiration complications were noted.

Conclusions: Fine needle aspiration cytology is safe and accurate in the diagnosis of tuberculosis and KS cervical lymphadenopathy among HIV-positive patients.
\end{abstract}

Keywords: Fine needle aspiration cytology, cervical lymphadenopathy, HIV

DOI: http://dx.doi.org/10.4314/ahs.v15i1.15

\section{Introduction}

Significant asymmetrical lymphadenopathy is a common problem in HIV infected adults. In developing countries with a high incidence of tuberculosis, tuberculous lymphadenitis (TBLN) is one of the most frequent causes of lymphadenopathy and occurs with increased frequency in HIV positive individuals. ${ }^{1-3}$ Several other opportunistic infections can present similar-

\section{Corresponding author: \\ Robert Kalyesubula \\ School of Medicine and School of \\ Biomedical Sciences, Makerere University \\ College of Health Sciences, Kampala, Uganda \\ Email: rkalyesubula@gmail.com / \\ rkalyes@yahoo.com}

ly including; Cryptococcus infection, toxoplasmosis and pyogenic infections. In HIV/AIDS patients, there is also an increased risk of development of malignancies such as Kaposi's sarcoma (KS) and non-Hodgkin's lymphoma (NHL) which may present similarly, ${ }^{4}$.

In the past decade, fine needle aspirate cytology (FNAC) has assumed an important role in the diagnosis of peripheral lymphadenopathy as an alternative procedure which is less invasive, faster and cheaper than excision biopsy. ${ }^{6-8}$ Unfortunately, FNAC has not been widely utilized in Uganda, as most clinicians still use surgical excision biopsies. Such biopsies are fairly invasive; need more resources, and a longer time to diagnosis. Diagnostic accuracy of FNAC with its pitfalls has been extensively reviewed by Schmidt. ${ }^{9}$ He argues that a set standard should be followed and in the design and reporting of these studies to avoid bias. FNAC has gen- 
erally been found to be accurate in diagnosing different lymph nodes, as well as height, weight, Karnosky score, aetiologies in HIV-infected patients but most of these and WHO HIV stage. Blood was drawn for CD4 T cell studies have been retrospective. ${ }^{9,10}$

count testing.

\section{The diagnostic accuracy of FNAC among HIV-infect- Test methods}

ed patients with cervical lymphadenopathy in Uganda Histology of the lymph node aspirate was used as the is largely unknown; neither do we know the leading oold standard for all the aetiological diaonoses. We percauses of lymp There is therefore need to ascertain the accuracy and causes of cervical lymphadenopathy and to evaluate simpler, less invasive methods for making a diagnosis. We hypothesized that FNAC is as good as histology in the diagnosis of lymphadenopathy among HIV-positive patients. We determined the diagnostic accuracy of FNAC in the evaluation of cervical lymphadenopathy in adult HIV-infected patients and established the common causes in Mulago national referral hospital.

\section{Methods}

\section{Participant}

This was a cross-sectional study of diagnostic accuracy in which we consecutively recruited adult HIV-infected patients with cervical lymphadenopathy who were admitted to Mulago national referral hospital medical wards in Kampala, Uganda, between February 2007 and June 2007. Mulago national referral hospital is one of two national referral hospitals. It is located in the center of the capital city of the country called Kampala. The hospital admits most of the HIV- infected patients from the surrounding specialist centers as well as other hospitals throughout the country. HIV testing is routinely provided to all the patients that are admitted through the emergency unit on an 'opt out' basis.

We included patients who were 18 years or older and had documented HIV-1 infection with cervical lymphadenopathy (considered as the largest lymph node diameter $>1$ centimeter). Patients with a prior histopathologic diagnosis of their cervical lympahadenopathy were excluded from the study.

We interviewed participants using a structured questionnaire to obtain information on the socio- demographic characteristics, history of prior treatment for TB or any other opportunistic infection as well as the presence of fever, cough for more than two weeks, night sweats and loss of weight.

Study physicians examined participants to assess the location, number and consistency of enlarged cervical

formed fine needle aspiration on the cervical lymph node of each participant. Under sterile conditions, we used a 23 -gauge needle and $10 \mathrm{ml}$ syringe for aspiration of the largest node, allowing 3-5 passes through the node. The aspirate was flushed onto a slide and spread to make a thin smear, taking precautions to avoid contamination especially to the eves.

Next local anesthetic was infiltrated in the skin above selected non-fluctuant lymph node that was easily accessible for biopsy. Under sterile conditions, the node was biopsied and put in a container with $10 \%$ formalin for histological processing and examination. Any discomfort or side effects were monitored over the nex four hours. The time taken to perform the FNA, biopsy and cytology were recorded.

The aspirated material was spread on five glass slides. One slide was fixed immediately with $95 \%$ ethyl alcohol, and the rest were air dried. The alcohol fixed slide was stained by haematoxylin and eosin (H\&E) stain fo cytological examination. For the air dried slides, one was for Ziehl Neelsen (ZN) staining to look for Acid Alcohol Fast Bacilli (AAFB) and the other slides were stained by Diff Quick stain for cytological examination at a later time. Cytology and histology was performed by a senior and experienced pathologist who has been doing similar work using the set criteria outlined below. For each of the specimen AAFB microscopy and with blinding to the first set of results. In each case a summary of clinical information was made available to the pathologist.

For quality control $10 \%$ of the specimen were randomly selected and reviewed by an independent senior pathologist. Histological diagnosis of TB was made basing on at least one of the three histological findings including: caseous necrosis, fibrosis or granulomas." Diagnosis of KS was based on finding clusters or single spindle cells and or plump cells in a back ground of numerous red blood cells and mature lymphocytes. ${ }^{12}$ The Reed Sternberg cells and "popcorn" cells formed the basis for diagnosis of Hodgkin's disease. Reactive adeni- tis was based on finding mixed population of lymphoid Ethical approval

cells on histology of the lymph node tissue. The cyto- The study was approved by the Faculty of Medicine ogical diannosis of toxoplasmosis was made based on Research and Ethics Committee of the School of Medseeing tangible body macrophages and a background of icine, Makerere University College of Health Sciences mature lymphocytes, predominantly small lymphocytes. and the Uganda National Council for Science and Tech-

\section{Statistical analysis} nology and written informed consent was obtained

All data was double entered into Epi-Info version 6.04 and analysed using SPSS 10.0 software.

from all study participants. cytology were done first and later histology was done
Descriptive statistics were used to summarize baseline Between February 2007 and June 2007, 1286 patient characteristics of the study patients, into means, medi- with HIV infection were admitted to medical wards ans, proportions, and standard deviations. The diagnos- at Mulago National Referral Hospital in Kampala and tic yield of the 2 procedures was compared by diagno- screened for enrolment (see figure 1). Among the ensis. To determine the diagnostic accuracy of FNAC in rolled 108 patients, the mean age was 33 years (Range the evaluation of aetiology of cervical lymphadenop- $18-60$ years) and $59 \%$ were men. Thirty nine patients athy; we estimated the sensitivity, specificity; negative $(36.2 \%)$ had generalized lymphadenopathy while sixty and positive predictive values using histology as the nine (63.9) had only cervical lymphadenopathy.

gold standard.

\section{Figure 1 Patient Flow Chart}

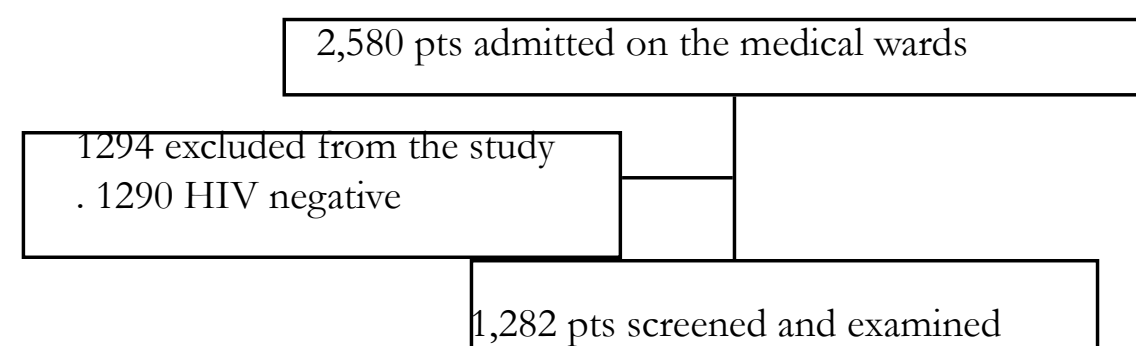

1,282 pts screened and examined

$1,1 / 4$ pts excludec

173 No significant lymphadenopathy

1 had prior cervical histopathological exam

108pts with significant lymphadenopathy

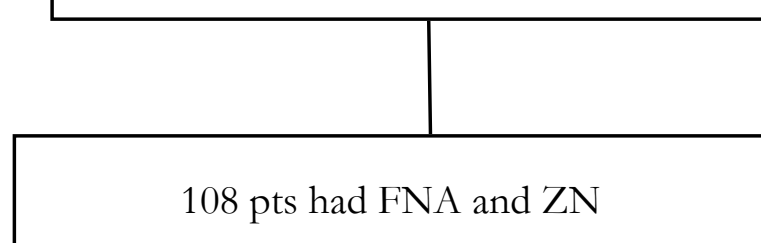

73 pts had biopsy done

Abbreviations: HIV, Human Immunodeficiency virus; Pts-patients, ZN, Zeibl Neelsen stain 
Seventy four patients (68.5\%) had tested for HIV with- a Karnofsky performance status of less than $50 \%$ and in the past one year and with $107(99.1 \%)$ presenting as only 62(57.4\%) were receiving cotrimoxazole prophyadvanced WHO HIV stage III and IV. The mean CD4 laxis (table 1). Only $22(20 \%)$ were receiving ART, with count for the study patients was 83 (range, 22-375) stavudine, lamuvidine and nevirapine (75\%) commoncells $/ \mathrm{mm} 3$. Up to $106(98.1 \%)$ were quite ill with est ART combination according to the guidelines at that time.

\section{Table 1: Baseline characteristics of study participants with cervical lymphadenopathy in Uganda}

\begin{tabular}{ll}
\hline Characteristic & $\mathrm{N}=108$ \\
\hline Men, number (\%) & $64(59.3)$ \\
Mean age in years, mean (range) & $33(18-60)$ \\
Mean BMI, $\mathrm{Kg} / \mathrm{m}^{2}$, mean (range) & $23.7(17-34)$ \\
Lymphadenopathy, number (\%) & \\
Cervical only & $69(63.9)$ \\
Generalized & $34(36.1)$ \\
Respiratory examination, number (\%) & \\
Normal & $87(80.6)$ \\
Abnormal (crackles/pleural effusion) & $21(19.4)$ \\
Period since diagnosis of HIV, number (\%) & \\
$<1$ year & $74(68.5)$ \\
1-5years & $27(25.0)$ \\
$>5 y$ years & $7(6.5)$ \\
WHO stage, number (\%) & \\
Stage 1 \&2 & $1(0.9)$ \\
Stage 3\&4 & $107(99.1)$ \\
CD4 cells/mm ${ }^{3}$, mean (range) & $83(22-375)$ \\
Receiving cotrimoxazole, number (\%) & $62(57.4)$ \\
Receiving ART, number (\%) & $22(20.0)$ \\
Karnofsky score, <50, number (\%) & $106(98.1)$ \\
\hline & \\
\hline & \\
\hline
\end{tabular}

Abbreviations: SD-standard deviation, BMI-Body mass index, ART- antiretroviral therapy

\section{Aetiology of cervical lymphadenopathy}

On FNAC diagnosis was $65.7 \%$ tuberculosis, $10.2 \%$ Out of 108 patients, only 73 had both fine needle as- Kaposi's sarcoma, 7.4\% non specific reactive adenitis, piration and biopsy done, while $35(32.4 \%)$ had only $3.7 \%$ Hodgkin's lymphoma and one patient each $0.9 \%$ cessible for biopsy because they were too deep or were ble 2). Diagnosis by histology showed; $79.5 \%$ tuberfluctuant. culosis, $13.6 \%$ Kaposi's sarcoma, $4.1 \%$ non specific reactive adenitis, $1.3 \%$ non Hodgkin's lymphoma and
$1.3 \%$ secondary metastatic carcinoma (table 2). FNAC itive ZN. KS was seen at a significantly higher CD4 was not able to come up with a conclusive diagnosis in $(153, \mathrm{SD} 177)$ cells $/ \mathrm{mm} 3$ as compared to tuberculosis 9.3\% of the samples obtained. Among the 58 patients whose mean CD4 cell counts was 70 (SD, 97) cells/ with histological diagnosis of TB, only $35(60.3 \%)$ had $\mathrm{mm} 3$ (p-value, 0.034$)$. None of the selected samples for a positive ZN on FNA and among the 71 patients with quality control $(10 \%)$ had a disagreement with the origcytological diagnosis of TB, only $48(67.6 \%)$ had a pos- inal diagnosis.

Table 2. Diagnosis of cervical lymphadenopathy and mean CD4 among HIV-infected patients in Uganda

\begin{tabular}{|c|c|c|c|c|c|c|}
\hline \multirow[t]{2}{*}{ Diagnosis } & \multicolumn{3}{|l|}{ FNAC } & \multicolumn{3}{|c|}{ Histology } \\
\hline & Freq & $\%$ & $\begin{array}{l}\text { Mean CD4 } \\
\text { (+/-SD) }\end{array}$ & Freq & $\%$ & $\begin{array}{l}\text { Mean CD4 } \\
(+/-S D)\end{array}$ \\
\hline Tuberculosis & 71 & 65.7 & $68(100)$ & 58 & 79.5 & 70 (97) \\
\hline KS & 11 & 10.2 & 147(168) & 10 & 13.6 & 153 (177) \\
\hline Reactive & 8 & 7.4 & $114(92)$ & 3 & 4.1 & $57(65)$ \\
\hline $\mathrm{HD}$ & 4 & 3.7 & $113(88)$ & 0 & 0 & NA \\
\hline NHL TOXO & 1 & 0.9 & NA & 1 & 1.3 & NA \\
\hline Metastatic ca. & 1 & 0.9 & NA & 0 & 0 & NA \\
\hline Non diagnostic & 1 & 0.9 & NA & 1 & 1.3 & NA \\
\hline Missing results & 10 & 9.3 & 79 (79) & 0 & 0 & NA \\
\hline & 1 & 0.9 & NA & 0 & 0 & NA \\
\hline Total & 108 & 100 & & 73 & 100 & \\
\hline
\end{tabular}

Abbreviations: FNAC-Fine needle aspiration cytology; HD-Hodgkin's disease;
KS-Kaposis Sarcoma;NA-Not applicable; NHL-Non Hodgkin's Lymphoma; Toxo-toxoplasmosis

Plates A to D (figure 2) show the histological diagnosis smear. All patients had caseous necrosis and 46 of the four commonest causes of cervical lymphad- (64.8) had granulomas.

enopathy. Of the 71 patients diagnosed with TB on Seventy seven percent of the patients with lymphadeFNAC, only 48 patients $(67.6 \%)$ had a positive ZN nopathic KS had mucocutaneous lesions. 


\section{Figure 2 : FNAC PLATES}

A FNAC of $\mathrm{c}$ er vi $\mathrm{c}$ al $l \mathrm{ymp}$ h nod e $\mathrm{f} \mathrm{r}$ om a 28 year ol d femal e show i ng ep i thel oi d c el 1 gr anul omas (w hi te ar r ow s) and c aseous nec rosis (bl ack ar r ow s). (Di f f Quick stai n. Mag ni f i c at i on x 40).

B FNAC f r om c er vi c al l ymp h nod e of a 24 year ol d mal e show i ng c l ust er s of sp i nd le c ell s (w hi t e ar r ow s). (H\& E st ai n. Mag ni f i c at i on x 40).

C FNAC of a c er vi c al l ymp h nod e show i ng Reed -St er nb er g c el $1 \mathrm{~s}$ (w hi t e ar r ow) and p op c or n c el $\mathrm{ls}$ (bl ac k ar r ow ) f r om a 26 year ol d femal e p at i ent w it h Hod g k i n's d i sease. (H\& E st ai n. Mag ni $f$ ic at $i$ on $x$ 40).

D FNAC $\mathrm{f} r$ om a c er vi c al $l$ ymp h nod e of a 26 year ol d HIV p osi t $i$ ve femal e $\mathrm{p}$ at $\mathrm{i}$ ent w $\mathrm{ith}$ a r eac $\mathrm{t} i$ ve nod e. Not e t he d if fer ent st ages of 1 ymp hoc yt e mat ur at i on (ar r ow s). (Di f f Quick st ai n. Mag ni $\mathrm{f}$ i c at i on $\mathrm{x}$ 40) FNAC-f i ne need $\mathrm{l}$ e asp i $\mathrm{r}$ at e c yt ol og y; H\& E- Hemat oxyli n and Eosi n

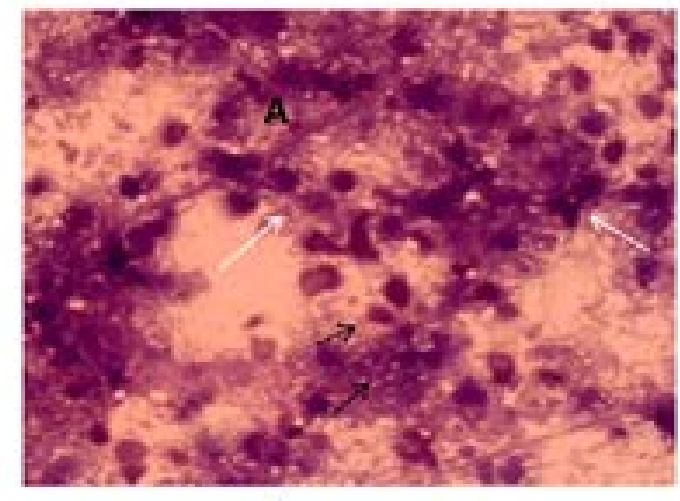

C

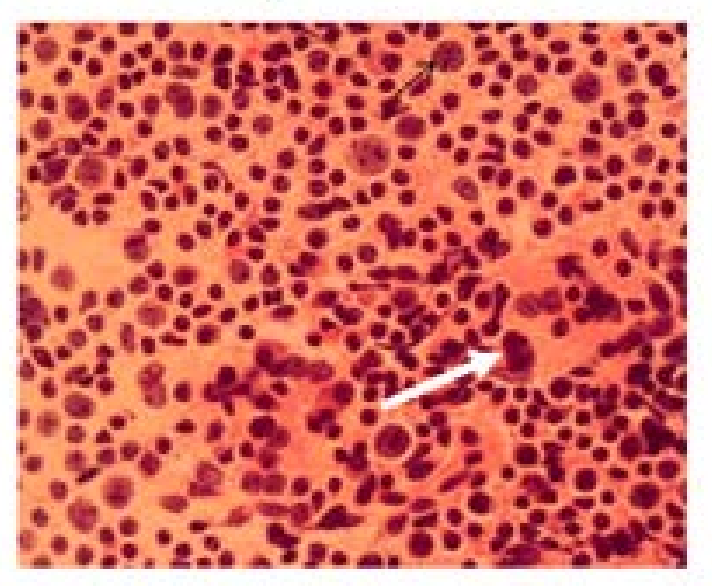

Duration and complications of FNA procedure The FNA procedure was shorter to perform as compared to the biopsy. FNA took a maximum of five minutes to be completed before processing. Processing the slide took another average of ten minutes in the ward side laboratory. We recorded no immediate complications with the FNA and none was reported in the period between doing the procedure and returning of the patients results. To carry out an open lymph node biopsy required surgical instruments, local anaesthetic agent, surgical sutures and draping towels. The minimum time which was taken to complete an open lymph
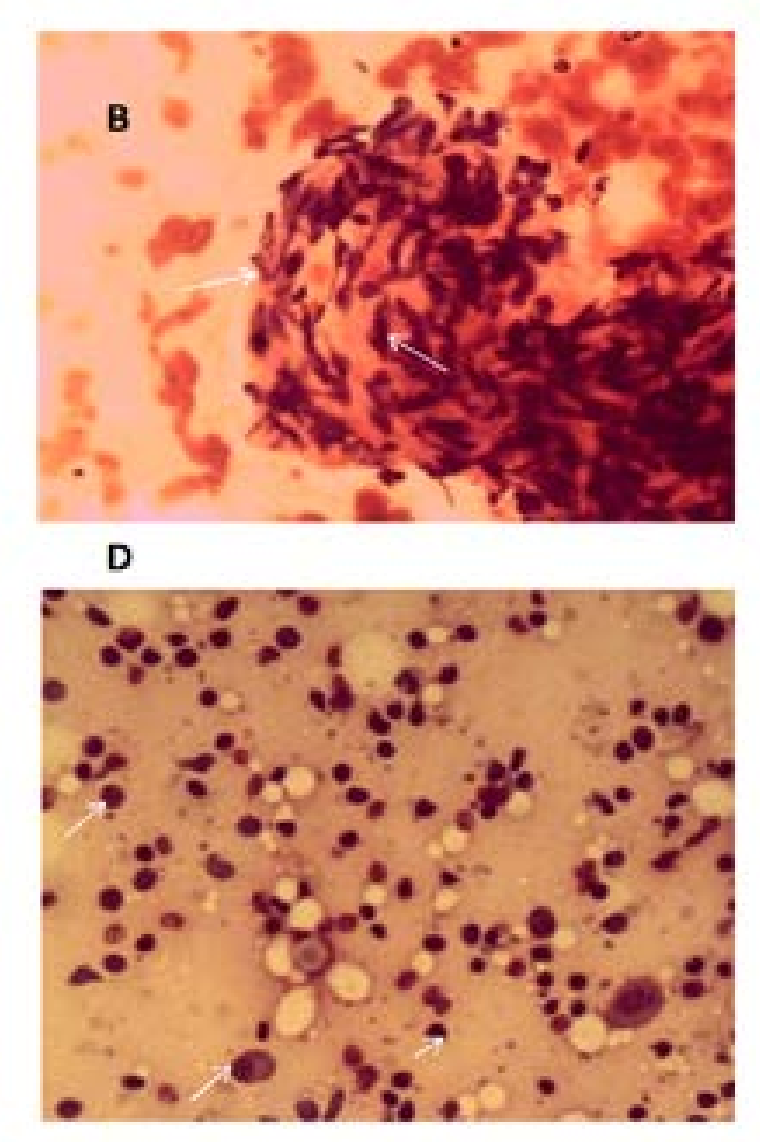

node biopsy was 20 minutes and processing the specimen required a minimum time of 24 hours. However no immediate complications were reported by any $\mathrm{pa}$ tient following the biopsies from the time of biopsy to the time of returning the histology results.

\section{Diagnostic accuracy of FNAC}

FNAC notably had a high diagnostic accuracy with a sensitivity of $93.1 \%$, specificity of $100 \%$, positive predictive value (PPV) of $100 \%$ and negative predictive (NPV) value of $79.9 \%$ for TB and sensitivity of $80 \%$ specificity of $98.4 \%$, PPV of $88.9 \%$ and NPV of $98.9 \%$

for KS when compared to histology. However, the di- $\quad 66.7 \%$ and a Kappa score of 0.65 for the diagnosis of agnostic accuracy was relatively low with a sensitivity of reactive adenitis (table 3).

Table 3. Sensitivity, specificity, PPV, NPV, kappa statistics of FNAC compared to histology among HIVinfected patients in Mulago Hospital, Uganda

\begin{tabular}{llllll}
\hline Diagnosis & Sensitivity (\%) & Specificity (\%) & PPV (\%) & NPV (\%) & Kappa score \\
\hline TB & 93.1 & 100 & 100 & 78.9 & 0.85 \\
Reactive & 66.7 & 98.5 & 66.7 & 98.5 & 0.65 \\
KS & 80 & 98.4 & 88.9 & 96.8 & 0.82
\end{tabular}

$\overline{T B-t u b e r c u l o s i s, ~ K S-K a p o s i ' s ~ s a r c o m a, ~ P P V-p o s i t i v e ~ p r e d i c t i v e ~ v a l u e, ~ N P V-n e g a t i v e ~ p r e d i c t i v e ~ v a l u e ~}$

Overall, 65(89\%) of FNAC findings were in agreement with the histology results for the same patients. For the other diagnoses the numbers were too small, to determine the accuracy of FNAC. The non diagnostic FNACs were 10 and the main reason for these was insufficient sample material from FNA according to the pathologist. These non diagnostic FNAs were encountered in instances where a bloody aspirate was got. Two of these bloody stained aspirates, later turned out to be KS on lymph node biopsy. The remaining eight non diagnostic FNAs, a diagnosis was not made at all, since even the biopsy was not done due to inaccessibility of the nodes. One patient had both TB and KS found in one lymph node on FNAC.

\section{Discussion}

Diagnostic accuracy

Overall FNAC diagnosis was in agreement with histological diagnosis in more than four fifth of the patients, which is quite high and shows that FNAC is generally a very accurate diagnostic procedure in HIV-infected patients with cervical lymphadenopathy. This was comparable to earlier studies done elsewhere. ${ }^{13,14}$ FNAC was able to come up with a diagnosis in $83 \%(29 / 35) \mathrm{pa}-$ tients where biopsy was not possible, emphasizing the diagnostic utility of FNAC even when biopsy is difficult. $^{15}$

FNAC was found to be a highly accurate method in the diagnosis of $\mathrm{TB}$; with a sensitivity of over ninety percent and a specificity of $100 \%$. This compares favorably with other studies done elsewhere in the developing world where TB is endemic. ${ }^{10,16}$ These findings were different from studies done in the developed world where
TB is not endemic, where FNA was found to have a very low sensitivity of $46 \%$ in the diagnosis of TBLN. ${ }^{17}$ The $\mathrm{ZN}$ positive rate was over two thirds which falls within the reported range of $(10-77 \%))^{1,2,17}$ This emphasizes the fact that a negative $\mathrm{ZN}$ on FNA does not rule out tuberculosis.

There were no false positive diagnoses made on fine needle aspiration for the diagnosis of tuberculosis; however there were four false negatives when compared with histology the gold standard. This is comparable with what previous studies found. ${ }^{13,18}$ This study had a lower false negative rate in TB diagnosis when compared to a study by Aljafari et al in Sudan where he found a false negative rate of $38 \%$ using a bigger gauge needle. ${ }^{19}$ The difference, probably can be attributed to the differences in sizes of needles used, since the bigger the needle the higher the chances of haemorrhage distorting the cytological picture. There was an excellent kappa agreement of 0.85 between FNAC and histology results in the diagnosis of tuberculosis. This therefore means that results of FNAC are quite comparable to results of histology making it quite a useful method in diagnosis of TB adenitis even in the absence of histology. This therefore means that it will be a very useful method if employed for use for TB diagnosis in Uganda. FNAC was also highly accurate in the diagnosis of KS and this was comparable to what Bates et al found. ${ }^{18}$

Diagnosis of reactive adenopathy with FNAC had a relatively low sensitivity compared to other aetiologies like TB and KS. This clearly depicts the diagnostic pitfalls of FNAC in diagnosis of reactive adenopathy as also reported by Reid et al. ${ }^{20}$ The number of patients with 


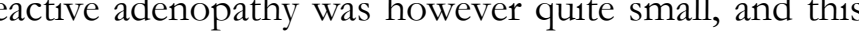
may have affected the result we got. Moreover, reactive adenitis has been questioned as a diagnosis ${ }^{21}$. For the other aetiologies of cervical lymph nodes found in our study, that is NHL and secondary metastatic carcinoma, the numbers were too small to determine the diagnostic accuracy of FNA in their diagnosis. One patient had both TB and KS found in one lymph node on FNAC. A similar finding was reported by Hayes in a suspected HIV patient case report. ${ }^{22}$ This re-emphasizes the possibility of two diagnoses in one node, especially in HIV positive patients as has been reported elsewhere. ${ }^{16,22}$ This dual aetiology however was discovered on FNAC in this study, and this was possible because more than one pass was made through the lymph nodes at different points.

Our study has several strengths. Our observations were drawn after screening over 1,200 patients in one of only two national referrals hospitals in Uganda. The hospital admits patients from most of the HIV care centers drawing a diverse population of HIV patients. We were unable to do lymph node biopsies in 35 patients who had cervical lymphadenopathy, since some lymph nodes were very deeply seated and not easily accessible for biopsy while in three patients, the nodes were fluctuant and not feasible for open biopsy. Biopsying a fluctuant lymph node carries a great risk of fistula formation. ${ }^{23,24}$ For such patients fine needle aspiration may be the only means of making the diagnosis and guide therapy with great accuracy according to our study. The majority of patients were below the fourth decade and this made the likelihood of finding other aetiologies less likely. In as much as this may be in agreement with the current demographics of HIV infection, these results may not be applicable to the older population of patients with HIV. Mycobacterial culture and PCR on the FNA material were not done for TB diagnosis. These may have improved on the diagnostic accuracy of FNAC and should be included in future studies.

\section{Aetiology of cervical lymphadenopathy}

Our study found tuberculosis to be the commonest cause of cervical lymphadenopathy among the stud patients. This was similar to what was found in previous studies done in Uganda and other developing countries. ${ }^{25-27}$ The percentage of TB was higher in this study at $69.4 \%$ as compared to Adrigwe's study done in ulago hospital, where it was $47.5 \%$. This difference explained by the fact that this was a slightly different study population. Adrigwe studied a population of both HIV positives and negative patients, while only HIV positives were included in our study. It has been demonstrated that advanced HIV infection may increase the diagnostic vield of FNA. This has been attributed to reduced immunological capacity of the immune system to clear off the mycobacterium in HIV infected patients.

The second commonest cause of cervical lymphadenopathy was Kaposi's sarcoma causing about a tenth of all adenopathies. This is comparable to the previous studies done. ${ }^{13,18,26}$ Over a third of patients with lymphadenopathic KS $(\mathrm{n}=10)$, had mucocutaneous $\mathrm{KS}$ as well, which emphasizes the importance of examination of the skin and mucous membranes in HIV positive patients with lymphadenopathy, since it may elucidate on the possible cause of lymphadenopathy.

Non specific reactive lymphadenopathy was the third commonest cause accounting for less than a tenth of all the lymphadenopathies. This was quite different from what Adrigwe found among medical patients with cervical lymphadenopathy, where this accounted for $(28.3 \%)$ of all aetiologies. ${ }^{26}$ Other studies elsewhere also differed greatly from the findings of our study, with reactive non specific adenitis occurring more frequently. ${ }^{10,13,17,28}$ Thi may be explained by the difference in the study populations, the patients in our study were probably more immune suppressed as compared to patients in other quoted studies above, making tuberculosis more likely than reactive adenitis to affect the nodes.

The majority of the patients were very sick with Karnofsky performance status of less than 50 . This may be because the study population constituted patients entirely from the medical wards, where patients are generally very sick and confined to bed in most cases. This shows that patients present late for care and may also explain the high mortality rate on the medical wards that has been reported in other studies. ${ }^{29,30}$

\section{Key messages}

FNAC is safe and accurate in the diagnosis of cervical lymphadenopathic tuberculosis and Kaposis sarcoma among HIV positive patients with advanced disease Tuberculosis is currently the commonest cause of cervical lymphadenopathy among HIV patients in Uganda.

FNAC is still useful in diagnosis of tuberculosis even when microscopy with Ziehl Neelsen (ZN) is negative

\section{Conclusion}

In summary, our study shows that tuberculosis is currently the commonest cause of cervical lymphadenopathy among HIV patients who participated in the study. FNAC is an accurate diagnostic method in evaluation of aetiology of cervical lymphadenopathy among HIV positive individuals, especially in the diagnosis of tuberculosis and Kaposi's sarcoma. Moreover, FNA is quite a useful diagnostic test even where lymph node biopsy is not possible. However, a negative FNA on ZN test does not rule out tuberculous adenitis and FNAC is still useful in diagnosis of tuberculosis even when $\mathrm{ZN}$ is negative.

\section{Acknowledgements}

This work was supported by the friends of Mengo United Kingdom and Mengo hospital management We thank Dr Kidaaga (cytological and histological), Mrs. Otwoda (processing and storage of histological specimen), Mr. A Andama (microscopy), Dr F Bwamabale and Ms L Nantayi (statistics), Ms S Nanziri and S Magara (data collection). We appreciate the study participants for their involvement and support during the study. Dr Kalyesubula is supported by grant by Mulago Foundation as the Rainer Arnhold Teaching Fellow.

\section{References}

1. Gupta AK, Nayar M, Chandra M. Critical appraisal of fine needle aspiration cytology in tuberculous lymphadenitis. Acta Cytol. May-Jun 1992;36(3):391-394 2. Finfer M, Perchick A, Burstein DE. Fine needle aspiration biopsy diagnosis of tuberculous lymphadenitis in patients with and without the acquired immune deficiency syndrome. Acta Cytol. May-Jun 1991;35(3):325 332.

3. Shafer RW, Kim DS, Weiss JP, Quale JM. Extrapulmonary tuberculosis in patients with human immunodeficiency virus infection. Medicine (Baltimore). Nov 1991;70(6):384-397.

4. Newton R, Grulich A, Beral V, etal. Cancer and HIV infection in Rwanda. Lancet. May 27 1995;345(8961):1378 1379.

5. Sitas F, Bezwoda WR, Levin V, et al. Association be- ween human immunodeficiency virus type 1 infection and cancer in the black population of Johannesburg and Soweto, South Africa. Br I Cancer. 1997;75(11):1704 1707

6. Carter TR, Feldman PS, Innes DJ, Jr., Frierson HF, Jr., Frigy AF. The role of fine needle aspiration cytology in the diagnosis of lymphoma. Acta Cytol. Nov-Dec 1988;32(6):848-853.

7. Tani EM, Christensson B, Porwit A, Skoog L. Immunocytochemical analysis and cytomorphologic diagnosis on fine needle aspirates of lymphoproliferative disease. Acta Cytol. Mar-Apr 1988;32(2):209-215.

8. Jimenez-Heffernan JA, Vicandi B, Lopez-Ferrer P, Hardisson D, Viguer JM. Value of fine needle aspiration cytology in the initial diagnosis of Hodgkin's disease. Analysis of 188 cases with an emphasis on diagnostic pitfalls. Acta Cytol. May-Jun 2001;45(3):300-306.

9. Schmidt RL, Witt BL, Lavfield LJ. Quality appraisal of diagnostic accuracy studies in fine-needle aspiration cytology: a survey of risk of bias and comparability. Arch Pathol Lab Med. 2013;137(4):566-575.

10. el Hag IA, Chiedozi LC, al Reyees FA, Kollur SM. Fine needle aspiration cytology of head and neck masses. Seven years' experience in a secondary care hospital. Acta Cytol. May-Jun 2003;47(3):387-392.

1. Knox J, Lane G, Wong JS, Trevan PG, Karunajeewa H. Diagnosis of Tuberculous Lymphadenitis Using Fine Needle Aspiration Biopsy. Intern Med J. Feb 28 2012.

12. Daskalopoulou D, Galanopoulou A, Statiropoulou P, Papapetrou S, Pandazis I, Markidou S. Cytologically interesting cases of primary skin tumors and tumor-like conditions identified by fine-needle aspiration biopsy. Diagn Cytopathol. Jul 1 1998;19(1):17-28.

13. Bottles K, McPhaul LW, Volberding P. Fine-needle aspiration biopsy of patients with acquired immunodeficiency syndrome (AIDS): experience in an outpatient clinic. Ann Intern Med. Jan 1988;108(1):42-45.

14. Strigle SM, Martin SE, Levine AM, Rarick MU. The use of fine needle aspiration cytology in the management of human immunodeficiency virus-related non-Hodgkin's lymphoma and Hodgkin's disease. J Acquir Immune Defic Syndr. Dec 1993;6(12):1329-1334.

15. Tachibana T, Orita Y, Fujisawa M, et al. Factors that make it difficult to diagnose cervical tuberculous lymphadenitis. J Infect Chemother. Jul 102013.

16. Strigle SM, Rarick MU, Cosgrove MM, Martin SE. A review of the fine-needle aspiration cytology findings in human immunodeficiency virus infection. Diagn $\mathrm{Cy}$ topathol. 1992;8(1):41-52. 
17. Ellison E, Lapuerta P, Martin SE. Fine needle aspiration diagnosis of mycobacterial lymphadenitis. Sensitivity and predictive value in the United States. Acta Cytol. Mar-Apr 1999;43(2):153-157.

18. Martin-Bates E, Tanner A, Suvarna SK, Glazer G, Coleman DV. Use of fine needle aspiration cytology for investigating lymphadenopathy in HIV positive patients. J Clin Pathol. Jun 1993;46(6):564-566.

19. Aljafari AS, Khalil EA, Elsiddig KE, et al. Diagnosis of tuberculous lymphadenitis by FNAC, microbiological methods and PCR: a comparative study. Cytopathology. Feb 2004;15(1):44-48.

20. Reid AJ, Miller RF, Kocjan GI. Diagnostic utility of fine needle aspiration (FNA) cytology in HIV-infected patients with lymphadenopathy. Cytopathology. Aug 1998;9(4):230-239.

21. Kalungi S WH, Bostad L. Reactive lymphadenopathy in Ugandan patients and its relationship to EBV and HIV infection. APMIS. 2009;117(4):302-307.

22. Hayes MM, Coghlan PJ, King H, Close P. Kaposi's sarcoma, tuberculosis and Hodgkin's lymphoma in a lymph node--possible acquired immunodeficiency syndrome. A case report. $S$ Afr Med J. Aug 11 1984;66(6):226-229.

23. Fontanilla JM, Barnes A, von Reyn CF. Current diagnosis and management of peripheral tuberculous lymphadenitis. Clin Infect Dis. Sep 2011;53(6):555-562. 24. Martin-Blondel G, Mars LT, Liblau RS. Pathogenesis of the immune reconstitution inflammatory syndrome in HIV-infected patients. Curr Opin Infect Dis. Jun 2012;25(3):312-320.

25. Nambuya A, Sewankambo N, Mugerwa J, Goodgame R, Lucas S. Tuberculous lymphadenitis associated with human immunodeficiency virus (HIV) in Uganda. J Clin Pathol. Jan 1988;41(1):93-96.

26. Adrigwe J. Cervical lymphadenopathy in Ugandan adults at Mulago hospital a clinico pathological study. Kampala,1989.

27. Jha BC, Dass A, Nagarkar NM, Gupta R, Singhal S. Cervical tuberculous lymphadenopathy: changing clinical pattern and concepts in management. Postgrad Med J. Mar 2001;77(905):185-187.

28. Patil PS, Bem C. Wide needle aspiration cytology in the diagnosis of lymphadenopathy in Zambia. J Clin Pathol. Sep 1993;46(9):806-809.

29. Kyeyune R, den Boon S, Cattamanchi A, et al. Causes of early mortality in HIV-infected TB suspects in an East African referral hospital. J Acquir Immune Defic Syn$d r$. Dec 2010;55(4):446-450.

30. Cox JA, Lukande RL, Nelson AM, et al. An autopsy study describing causes of death and comparing clinico-pathological findings among hospitalized patients in Kampala, Uganda. PLoS One. 2012;7(3):e33685. 